\title{
Dispersion of ratio block sequences and asymptotic density
}

\author{
by
}

\author{
Ferdinánd Filip (Komárno), Ladislav Mišík (Ostrava) and \\ JÁNos T. Tóth (Ostrava)
}

1. Introduction. We first recall some basic definitions. Denote by $\mathbb{N}$ and $\mathbb{R}^{+}$the sets of all positive integers and positive real numbers, respectively. For $X \subset \mathbb{N}$ let $X(n)=\#\{x \in X: x \leq n\}$. In the whole paper we will assume that $X$ is infinite. Denote by $R(X)=\{x / y: x \in X, y \in X\}$ the ratio set of $X$ and say that a set $X$ is $(R)$-dense if $R(X)$ is (topologically) dense in $\mathbb{R}^{+}$. The concept of $(R)$-density was defined and first studied in [10] and [11]. The quantities

$$
\begin{gathered}
\underline{d}(X)=\liminf _{n \rightarrow \infty} \frac{X(n)}{n}, \quad \bar{d}(X)=\limsup _{n \rightarrow \infty} \frac{X(n)}{n}, \\
d(X)=\lim _{n \rightarrow \infty} \frac{X(n)}{n}
\end{gathered}
$$

are the lower asymptotic density, upper asymptotic density, and asymptotic density (if defined), respectively. Relations between $(R)$-density, asymptotic density and logarithmic density have been studied, among others, in [5], [6], [14] and [15].

Now let $X=\left\{x_{1}, x_{2}, \ldots\right\}$ where $x_{n}<x_{n+1}$ are positive integers. Then

$$
\frac{x_{1}}{x_{1}}, \frac{x_{1}}{x_{2}}, \frac{x_{2}}{x_{2}}, \frac{x_{1}}{x_{3}}, \frac{x_{2}}{x_{3}}, \frac{x_{3}}{x_{3}}, \ldots, \frac{x_{1}}{x_{n}}, \frac{x_{2}}{x_{n}}, \ldots, \frac{x_{n}}{x_{n}}, \ldots
$$

is called the ratio block sequence of the set $X$. It is formed by the blocks $X_{1}, X_{2}, \ldots$, where

$$
X_{n}=\left(\frac{x_{1}}{x_{n}}, \frac{x_{2}}{x_{n}}, \ldots, \frac{x_{n}}{x_{n}}\right), \quad n=1,2, \ldots
$$

This kind of block sequences have been studied in [16], [18] and [1]. Also other kinds of block sequences have been studied by several authors (see [2],

2000 Mathematics Subject Classification: Primary 11B05.

Key words and phrases: dispersion, ratio block sequence, asymptotic density.

Supported by grants GAČR no. 201/04/0381, 201/07/0191 and VEGA no. 1/4006/07. 
[4], [7], [12] and [17]). Let $Y=\left(y_{n}\right)$ be an increasing sequence of positive integers. In [9], extending a result of [3], a sequence of blocks of the type

$$
Y_{n}=\left(\frac{1}{y_{n}}, \frac{2}{y_{n}}, \ldots, \frac{y_{n}}{y_{n}}\right)
$$

was investigated. The authors obtained a complete theory of uniform distribution of the related block sequence $\left(Y_{n}\right)$.

For every $n \in \mathbb{N}$ let

$$
D\left(X_{n}\right)=\max \left\{\frac{x_{1}}{x_{n}}, \frac{x_{2}-x_{1}}{x_{n}}, \ldots, \frac{x_{i+1}-x_{i}}{x_{n}}, \ldots, \frac{x_{n}-x_{n-1}}{x_{n}}\right\}
$$

be the maximum distance between two consecutive terms in the $n$th block. We will consider the quantity (see [18])

$$
\underline{D}(X)=\liminf _{n \rightarrow \infty} D\left(X_{n}\right),
$$

called the dispersion of the block sequence (1) derived from $X$, and its relations to the previously mentioned asymptotic density of the original set $X$.

To end this section, let us mention the concept of dispersion of a general sequence of numbers in the interval $[0,1]$. Let $\left(x_{n}\right)_{n=0}^{\infty}$ be a sequence in $[0,1]$. For every $N \in \mathbb{N}$ let $x_{i_{1}} \leq \cdots \leq x_{i_{N}}$ be a nondecreasing rearrangement of its first $N$ terms and define

$$
d_{N}=\frac{1}{2} \max \left\{\max \left\{x_{i_{j+1}}-x_{i_{j}}: j=1, \ldots, N-1\right\}, x_{i_{1}}, 1-x_{i_{N}}\right\}
$$

to be the dispersion of the finite sequence $x_{0}, x_{1}, \ldots, x_{N}$. The properties of this concept can be found for example in [8] where it is also proved that

$$
\limsup _{N \rightarrow \infty} N d_{N} \geq \frac{1}{\log 4}
$$

for every one-to-one infinite sequence $x_{n} \in[0,1)$. Notice that the density of the whole sequence $\left(x_{n}\right)_{n=0}^{\infty}$ is equivalent to $\lim _{N \rightarrow \infty} d_{N}=0$. The dispersion of block sequences defined in the present paper does not have the analogous property. Much more on these and related topics can be found in [13].

2. Results. When calculating $\underline{D}(X)$, the following theorems are often useful (see [18, Theorem 1 and Corollary 1]).

(A1) Let

$$
X=\left\{x_{1}, x_{2}, \ldots\right\}=\bigcup_{n=1}^{\infty}\left(c_{n}, d_{n}\right] \cap \mathbb{N},
$$

where $x_{n}<x_{n+1}$ and $c_{n}<d_{n}<c_{n+1}$, for $n \in \mathbb{N}$, are positive 
integers. Then

$$
\underline{D}(X)=\liminf _{n \rightarrow \infty} \frac{\max \left\{c_{i+1}-d_{i}: i=1, \ldots, n\right\}}{d_{n+1}} .
$$

(A2) Let $X$ be as in (A1). Suppose that there exists a positive integer $n_{0}$ such that

$$
c_{n+1}-d_{n} \leq c_{n+2}-d_{n+1} \quad \text { for all } n>n_{0} .
$$

Then

$$
\underline{D}(X)=\liminf _{n \rightarrow \infty} \frac{c_{n+1}-d_{n}}{d_{n+1}} .
$$

The following theorem relates the asymptotic density of a set $X \subset \mathbb{N}$ and the dispersion of the block sequence (1).

Theorem 1. Let $X \subset \mathbb{N}$. Then

(i) If $d(X)>0$ then $\underline{D}(X)=0$.

(ii) If $a=\underline{d}(X)<\bar{d}(X)=b$ then

$$
\underline{D}(X) \leq \frac{(1-b)(b-a)}{b(1-a)} .
$$

(iii) If $d(X)=0$ then $\underline{D}(X)$ can be any number in the interval $[0,1]$.

Proof. Write $X=\bigcup_{n=1}^{\infty}\left(c_{n}, d_{n}\right] \cap \mathbb{N}$ and define $h(n)=X(n) / n$ for $n \in \mathbb{N}$. For the purposes of this proof we introduce the following notation. For every $n \in \mathbb{N}$ let $\mu(n)$ be the smallest integer in $\{1, \ldots, n\}$ such that

$$
c_{\mu(n)+1}-d_{\mu(n)}=\max \left\{c_{i+1}-d_{i}: i=1, \ldots, n\right\} .
$$

Notice that in this notation (A1) says that

$$
\underline{D}(X)=\liminf _{n \rightarrow \infty} \frac{c_{\mu(n)+1}-d_{\mu(n)}}{d_{n+1}} .
$$

Now there are two possibilities:

(a) $\mu=\lim _{n \rightarrow \infty} \mu(n)<\infty$,

(b) $\mu=\lim _{n \rightarrow \infty} \mu(n)=\infty$.

In case (a), (2) clearly yields $\underline{D}(X)=0$ and the statement of the theorem holds. Thus in the following we assume that (b) holds.

By definition of the upper asymptotic density, for every $p \in \mathbb{N}$ there exists a $k=k(p) \in \mathbb{N}$ such that $k(p) \rightarrow \infty$ as $p \rightarrow \infty$ and

$$
h\left(d_{k+1}\right)>\bar{d}(X)-1 / p \text {. }
$$

To simplify the writing, set

$$
c_{\mu(k)+1}=n=n(p) ; \quad c_{\mu(k)+1}-d_{\mu(k)}=t=t(p) ; \quad d_{k+1}-c_{\mu(k)+1}=u=u(p) .
$$


Notice that $n, t, u$ are positive integers and

$$
h(n+u) \geq h(n) .
$$

Also, the conditions $n \rightarrow \infty$ and $p \rightarrow \infty$ are equivalent.

Using (A1) and observing that $\left\{d_{k(p)}\right\}_{p=1}^{\infty}$ is a subsequence of $\left\{d_{n}\right\}_{n=1}^{\infty}$ we find that

$$
\begin{aligned}
\underline{D}(X) & =\liminf _{n \rightarrow \infty} \frac{c_{\mu(n)+1}-d_{\mu(n)}}{d_{n+1}} \\
& \leq \liminf _{p \rightarrow \infty} \frac{c_{\mu(k(p))+1}-d_{\mu(k(p))}}{d_{k(p)+1}}=\liminf _{p \rightarrow \infty} \frac{t(p)}{n(p)+u(p)} .
\end{aligned}
$$

To upper bound the last fraction (notice that $X(n-t)=X(n)$ )

$$
\frac{t}{n+u}=\frac{n-(n-t)}{n+u}=\frac{\frac{X(n)}{h(n)}-\frac{X(n)}{h(n-t)}}{\frac{X(n)}{h(n)}+u},
$$

we estimate $u$ from below as follows. We have

$$
h(n+u)=\frac{X(n+u)}{n+u} \leq \frac{X(n)+u}{n+u},
$$

and consequently

$$
u \geq \frac{n h(n+u)-X(n)}{1-h(n+u)}
$$

Thus

$$
\begin{aligned}
\frac{t}{n+u} & \leq \frac{\frac{X(n)}{h(n)}-\frac{X(n)}{h(n-t)}}{\frac{X(n)}{h(n)}+\frac{n h(n+u)-X(n)}{1-h(n+u)}}=\frac{\frac{1}{h(n)}-\frac{1}{h(n-t)}}{\frac{1}{h(n)}+\frac{\frac{n h(n+u)}{X(n)}-1}{1-h(n+u)}} \\
& =\frac{\frac{1}{h(n)}-\frac{1}{h(n-t)}}{\frac{1}{h(n)}+\frac{\frac{h(n+u)}{h(n)}-1}{1-h(n+u)}}=\frac{\frac{h(n-t)-h(n)}{h(n) h(n-t)}}{\frac{1-h(n)}{h(n)(1-h(n+u))}} \\
& =(1-h(n+u)) \frac{h(n-t)-h(n)}{h(n-t)(1-h(n))} .
\end{aligned}
$$

(i) Suppose that $d(X)>0$. Then $\lim _{p \rightarrow \infty}(n(p)-t(p))=\infty$. Using the previously derived relations and taking into account (4), we have

$$
\begin{aligned}
\underline{D}(X) & \leq \liminf _{n \rightarrow \infty} \frac{1-h(n+u)}{1-h(n)} \frac{h(n-t)-h(n)}{h(n-t)} \\
& \leq \lim _{n \rightarrow \infty} \frac{h(n-t)-h(n)}{h(n-t)}=0 .
\end{aligned}
$$

The last equality follows from the fact that $n-t \rightarrow \infty$ as $p \rightarrow \infty$ and $d(X)=\lim _{m \rightarrow \infty} h(m)>0$. 
(ii) Let $a=\underline{d}(X)<\bar{d}(X)=b$. Then

$\lim _{p \rightarrow \infty} h(n(p)+u(p))=b, \quad \liminf _{p \rightarrow \infty} h(n(p)) \geq a, \quad \limsup _{p \rightarrow \infty} h(n(p)-t(p)) \leq b$.

A simple analysis of the last term in (6) shows that it is increasing in $h(n-t)$ and decreasing in $h(n)$. Thus, by (5), (6) and taking into account (3), we have, for $n=n(p), u=u(p)$ and $t=t(p)$,

$$
\begin{aligned}
\underline{D}(X) & \leq\left(1-\lim _{p \rightarrow \infty} h(n+u)\right) \frac{\limsup _{p \rightarrow \infty} h(n-t)-\liminf _{p \rightarrow \infty} h(n)}{\limsup _{p \rightarrow \infty} h(n-t)\left(1-\liminf _{p \rightarrow \infty} h(n)\right)} \\
& \leq \frac{(1-b)(b-a)}{b(1-a)} .
\end{aligned}
$$

(iii) Let $\alpha \in(0,1)$. Then put $a=1 /(1-\alpha)>1$ and consider the set $X=\left\{\left[a^{n}\right]: n \in \mathbb{N}\right\}$. Then

$$
d(X)=\lim _{n \rightarrow \infty} \frac{n}{\left[a^{n}\right]}=0 .
$$

Moreover, $X$ satisfies the conditions of (A2) and so

$$
\underline{D}(X)=\liminf _{n \rightarrow \infty} \frac{\left[a^{n+1}\right]-1-\left[a^{n}\right]}{\left[a^{n+1}\right]}=\frac{a-1}{a}=\alpha .
$$

Now let $\alpha=0$. Then set $X=\left\{n^{2}: n \in \mathbb{N}\right\}$. One can easily show that $d(X)=0$ and, again using (A2), also $\underline{D}(X)=0=\alpha$.

Finally, let $\alpha=1$. Then set $X=\left\{2^{2^{n}}: n \in \mathbb{N}\right\}$. It can be easily shown that $d(X)=0$ and, once again by (A2), also $\underline{D}(X)=1=\alpha$.

Item (ii) of the above theorem implies that if $\bar{d}(X)=b=1$ then we immediately have $\underline{D}(X)=0$. In $[18$, Theorem 2] it is proved that $\underline{D}(X)=0$ implies density of the block sequence (1). This gives the result of [10] that $\bar{d}(X)=1$ implies $(R)$-density of $X$.

The following theorem says that the bound in (ii) is the best possible, as the set of values of the dispersion of sets with $\underline{d}(X)=a$ and $\bar{d}(X)=b$ is the whole interval $[0, \bar{\alpha}]$ where

$$
\bar{\alpha}:=\frac{(1-b)(b-a)}{b(1-a)} .
$$

TheOrem 2. Let $0 \leq a<b<1$ and $\alpha \in[0, \bar{\alpha}]$ with $\bar{\alpha}$ defined above. Then there exists a set $X \subseteq \mathbb{N}$ such that $\underline{d}(X)=a, \bar{d}(X)=b$ and $\underline{D}(X)=\alpha$.

Proof. First we consider the case $\alpha=\bar{\alpha}$. We have two possibilities: $a>0$ and $a=0$.

The case $a>0$. Let $0<a<b<1$. Choose a real number $d_{0}$ such that

$$
\min \left\{d_{0} \frac{b}{a} ; d_{0} \frac{1-a}{1-b}\right\}>d_{0}+1
$$


and define the sequences $\left\{c_{n}\right\}_{n=1}^{\infty}$ and $\left\{d_{n}\right\}_{n=1}^{\infty}$ as follows:

$$
c_{n}=\frac{b}{a} d_{n-1} \quad \text { and } \quad d_{n}=\frac{b(1-a)}{a(1-b)} d_{n-1} \quad \text { for all } n \in \mathbb{N} .
$$

Then (7) and (8) imply

$$
\begin{array}{cl}
{\left[c_{n}\right]<\left[d_{n}\right]<\left[c_{n+1}\right]} & \text { for all } n \in \mathbb{N}, \\
n^{2}=o\left(c_{n}\right) \quad \text { and } & n^{2}=o\left(d_{n}\right) .
\end{array}
$$

By (8) and (9), we can apply both statements (A1) and (A2) to the set $X \subset \mathbb{N}$ defined by

$$
X=\bigcup_{n=1}^{\infty}\left(\left[c_{n}\right],\left[d_{n}\right]\right] \cap \mathbb{N} .
$$

We are going to show that $\underline{d}(X)=a, \bar{d}(X)=b$ and $\underline{D}(X)=\bar{\alpha}$. From the definition of $X$, clearly

$$
\begin{aligned}
& \underline{d}(X)=\liminf _{n \rightarrow \infty} \frac{X\left(\left[c_{n}\right]\right)}{\left[c_{n}\right]}, \\
& \bar{d}(X)=\limsup _{n \rightarrow \infty} \frac{X\left(\left[d_{n}\right]\right)}{\left[d_{n}\right]} .
\end{aligned}
$$

From (8), (10) and (11) one can derive

$$
\begin{aligned}
\frac{X\left(\left[d_{n}\right]\right)}{\left[d_{n}\right]} & =\frac{\sum_{i=1}^{n}\left(\left[d_{i}\right]-\left[c_{i}\right]\right)}{\left[d_{n}\right]}=\frac{\sum_{i=1}^{n}\left(d_{i}-c_{i}\right)}{d_{n}}+o(1) \\
& =\frac{\sum_{i=1}^{n}\left(\left(\frac{b(1-a)}{a(1-b)}-\frac{b}{a}\right) d_{i-1}\right)}{d_{n}}+o(1)=\frac{b(b-a)}{a(1-b)} \sum_{i=1}^{n} \frac{d_{i-1}}{d_{n}}+o(1) \\
& =\frac{b(b-a)}{a(1-b)} \sum_{i=1}^{n}\left(\frac{a(1-b)}{b(1-a)}\right)^{i}+o(1) .
\end{aligned}
$$

The above relations yield

$$
\begin{aligned}
\bar{d}(X) & =\limsup _{n \rightarrow \infty} \frac{X\left(\left[d_{n}\right]\right)}{\left[d_{n}\right]}=\lim _{n \rightarrow \infty} \frac{X\left(\left[d_{n}\right]\right)}{\left[d_{n}\right]} \\
& =\frac{b(b-a)}{a(1-b)} \lim _{n \rightarrow \infty} \sum_{i=1}^{n}\left(\frac{a(1-b)}{b(1-a)}\right)^{i}=\frac{b(b-a)}{a(1-b)} \frac{\frac{a(1-b)}{b(1-a)}}{1-\frac{a(1-b)}{b(1-a)}}=b .
\end{aligned}
$$

Similarly, from (8), (14) and the definition of $X$ we obtain

$$
\begin{aligned}
\underline{d}(X) & =\liminf _{n \rightarrow \infty} \frac{X\left(\left[c_{n}\right]\right)}{\left[c_{n}\right]}=\liminf _{n \rightarrow \infty} \frac{\left[d_{n-1}\right]}{\left[c_{n}\right]} \frac{X\left(\left[c_{n}\right]\right)}{\left[d_{n-1}\right]} \\
& =\liminf _{n \rightarrow \infty} \frac{\left[d_{n-1}\right]}{\left[c_{n}\right]} \frac{X\left(\left[d_{n-1}\right]\right)}{\left[d_{n-1}\right]}=\frac{a}{b} b=a .
\end{aligned}
$$


By the definitions of $\left\{c_{n}\right\}_{n=1}^{\infty}$ and $\left\{d_{n}\right\}_{n=1}^{\infty}$,

$$
\left[c_{n+1}\right]-\left[d_{n}\right] \leq\left[c_{n+2}\right]-\left[d_{n+1}\right]
$$

for all sufficiently large $n \in \mathbb{N}$. By use of (A2) we obtain

$$
\underline{D}(X)=\liminf _{n \rightarrow \infty} \frac{\left[c_{n+1}\right]-\left[d_{n}\right]}{\left[d_{n+1}\right]}=\liminf _{n \rightarrow \infty} \frac{\frac{c_{n+1}}{d_{n}}-1}{\frac{d_{n+1}}{d_{n}}}=\frac{(1-b)(b-a)}{b(1-a)}=\bar{\alpha} .
$$

The case $a=0$. Let $0=a<b<1$. Choose $d_{0} \in \mathbb{R}^{+}$such that

$$
d_{0}>\frac{1-b}{b}
$$

Now define

$$
c_{n}=n d_{n-1} \quad \text { and } \quad d_{n}=\frac{n}{1-b} d_{n-1} \quad \text { for all } n \in \mathbb{N} .
$$

By (16) and (17), we see that (9) and (10) hold again, so we can apply (A1) and (A2) to

$$
X=\bigcup_{n=1}^{\infty}\left(\left[c_{n}\right],\left[d_{n}\right]\right] \cap \mathbb{N} .
$$

Again, we are going to show that $\underline{d}(X)=a, \bar{d}(X)=b$ and $\underline{D}(X)=\bar{\alpha}$. One can easily check the following bounds:

$$
\frac{\left[d_{n}\right]-\left[c_{n}\right]}{\left[d_{n}\right]} \leq \frac{X\left(\left[d_{n}\right]\right)}{\left[d_{n}\right]}=\frac{\sum_{i=1}^{n}\left(\left[d_{i}\right]-\left[c_{i}\right]\right)}{\left[d_{n}\right]} \leq \frac{\left[d_{n}\right]-\left[c_{n}\right]}{\left[d_{n}\right]}+\frac{\left[d_{n-1}\right]}{\left[d_{n}\right]}
$$

By (17) we have

$$
\lim _{n \rightarrow \infty} \frac{\left[d_{n}\right]-\left[c_{n}\right]}{\left[d_{n}\right]}=b \quad \text { and } \quad \lim _{n \rightarrow \infty} \frac{\left[d_{n-1}\right]}{\left[d_{n}\right]}=0 .
$$

Again, the definition of $X$ allows us to use (12) and (13) giving

$$
\begin{aligned}
& \bar{d}(X)=\limsup _{n \rightarrow \infty} \frac{X\left(\left[d_{n}\right]\right)}{\left[d_{n}\right]}=\lim _{n \rightarrow \infty} \frac{\left[d_{n}\right]-\left[c_{n}\right]}{\left[d_{n}\right]}=b, \\
& \underline{d}(X)=\liminf _{n \rightarrow \infty} \frac{X\left(\left[c_{n}\right]\right)}{\left[c_{n}\right]}=\liminf _{n \rightarrow \infty} \frac{\left[d_{n-1}\right]}{\left[c_{n}\right]} \frac{X\left(\left[d_{n-1}\right]\right)}{\left[d_{n-1}\right]}=\lim _{n \rightarrow \infty} \frac{1}{n} b=0 .
\end{aligned}
$$

The definitions of $\left\{c_{n}\right\}_{n=1}^{\infty}$ and $\left\{d_{n}\right\}_{n=1}^{\infty}$ imply (15) once more, so using (A2) we obtain

$$
\underline{D}(X)=\liminf _{n \rightarrow \infty} \frac{\left[c_{n+1}\right]-\left[d_{n}\right]}{\left[d_{n+1}\right]}=\liminf _{n \rightarrow \infty} \frac{n}{\frac{n+1}{1-b}}=1-b=\bar{\alpha},
$$

proving our statement in the case when $\alpha=\bar{\alpha}$.

The above sequences $\left\{c_{n}\right\}_{n \in \mathbb{N}}$ and $\left\{d_{n}\right\}_{n \in \mathbb{N}}$ and the set $X$ will also be used to prove the statement of our theorem for any $0 \leq \alpha<\bar{\alpha}$.

We will distinguish two cases: $\alpha>0$ and $\alpha=0$. 
Let $0<\alpha<\bar{\alpha}$. Define

$$
Z=\bigcup_{n=1}^{\infty}\left\{\left[d_{n}\right]+k\left[\frac{\alpha}{\bar{\alpha}}\left(\left[c_{n+1}\right]-\left[d_{n}\right]\right)\right]: k=1, \ldots, m_{n}\right\}
$$

where

$$
m_{n}\left[\frac{\alpha}{\bar{\alpha}}\left(\left[c_{n+1}\right]-\left[d_{n}\right]\right)\right] \leq\left[c_{n+1}\right]-\left[d_{n}\right]<\left(m_{n}+1\right)\left[\frac{\alpha}{\bar{\alpha}}\left(\left[c_{n+1}\right]-\left[d_{n}\right]\right)\right] .
$$

First we show that $d(Z)=0$. To see this, let $x \in \mathbb{N}$ and $\left[c_{n}\right]<x \leq\left[c_{n+1}\right]$. Then, using (10) and (18), we obtain

$$
0 \leq \frac{Z(x)}{x} \leq \frac{\sum_{i=1}^{n} m_{i}}{\left[c_{n}\right]}=O\left(\frac{\frac{\bar{\alpha}}{\alpha} n}{\left[c_{n}\right]}\right)=o\left(\frac{1}{n}\right)
$$

which yields $d(Z)=0$.

Set $Y=X \cup Z$. As $d(Z)=0$, we have

$$
\underline{d}(Y)=\underline{d}(X)=a \quad \text { and } \quad \bar{d}(Y)=\bar{d}(X)=b .
$$

It remains to show that $\underline{D}(Y)=\alpha$.

By definition of $Y$, we can apply (A1) to obtain

$$
\underline{D}(Y)=\liminf _{n \rightarrow \infty} \frac{\left[\frac{\alpha}{\bar{\alpha}}\left(\left[c_{n+1}\right]-\left[d_{n}\right]\right)\right]}{\left[d_{n+1}\right]}=\frac{\alpha}{\bar{\alpha}} \bar{\alpha}=\alpha .
$$

Now let $\alpha=0$. Define

$$
Z=\bigcup_{n=1}^{\infty}\left\{\left[d_{n}\right]+k\left[\frac{\left[c_{n+1}\right]-\left[d_{n}\right]}{n}\right]: k=1, \ldots, n\right\} .
$$

Again $d(Z)=0$, as for every $x \in \mathbb{N}$ with $\left[c_{n}\right]<x \leq\left[c_{n+1}\right]$ we have

$$
\frac{Z(x)}{x} \leq \frac{\sum_{i=1}^{n} i}{\left[c_{n}\right]}=O\left(\frac{n^{2}}{\left[c_{n}\right]}\right)=o(1) .
$$

Put $Y=X \cup Z$. We have immediately

$$
\underline{d}(Y)=\underline{d}(X)=a \text { and } \bar{d}(Y)=\bar{d}(X)=b .
$$

By definition of $Y$, we can apply (A1) to obtain

$$
\underline{D}(Y)=\liminf _{n \rightarrow \infty} \frac{\left[\frac{1}{n}\left(\left[c_{n+1}\right]-\left[d_{n}\right]\right)\right]}{\left[d_{n+1}\right]}=\lim _{n \rightarrow \infty} \frac{1}{n} \bar{\alpha}=0
$$

finishing the proof.

\section{References}

[1] F. Filip and J. T. Tóth, On estimations of dispersions of certain dense block sequences, Tatra Mt. Math. Publ. 31 (2005), 65-74.

[2] E. Hlawka, The Theory of Uniform Distribution, AB Academic Publishers, London, 1984. 
[3] S. Knapowski, Über ein Problem der Gleichverteilung, Colloq. Math. 5 (1958), 8-10.

[4] L. Kuipers and H. Niederreiter, Uniform Distribution of Sequences, Wiley, New York, 1974.

[5] L. Mišík, Sets of positive integers with prescribed values of densities, Math. Slovaca 52 (2002), 289-296.

[6] L. Mišík and J. T. Tóth, Logarithmic density of a sequence of integers and density of its ratio set, J. Théor. Nombres Bordeaux 15 (2003), 309-318.

[7] G. Myerson, A sampler of recent developments in the distribution of sequences, in: Number Theory with an Emphasis on the Markoff Spectrum (Provo, UT, 1991), Lecture Notes in Pure Appl. Math. 147, Dekker, New York, 1993, 163-190.

[8] H. Niederreiter, On a measure of denseness for sequences, in: Topics in Classical Number Theory (Budapest, 1981), Vols. I, II, G. Halász (ed.), Colloq. Math. Soc. János Bolyai 34, North-Holland, Amsterdam, 1984, 1163-1208.

[9] Š. Porubský, T. Šalát and O. Strauch, On a class of uniformly distributed sequences, Math. Slovaca 40 (1990), 143-170.

[10] T. Šalát, On ratio sets of sets of natural numbers, Acta Arith. 15 (1969), 273-278.

[11] -, Quotientbasen und $(R)$-dichte Mengen, ibid. 19 (1971), 63-78.

[12] I. J. Schoenberg, Über die asymptotische Verteilung reeller Zahlen mod 1, Math. Z. 28 (1928), 171-199.

[13] O. Strauch and Š. Porubský, Distribution of Sequences: A Sampler, Peter Lang, Frankfurt am Main, 2005.

[14] O. Strauch and J. T. Tóth, Asymptotic density of $A \subset \mathbb{N}$ and density of the ratio set $R(A)$, Acta Arith. 87 (1998), 67-78.

[15] - - - Corrigendum to Theorem 5 of the paper "Asymptotic density of $A \subset \mathbb{N}$ and density of the ratio set $R(A)$ " (Acta Arith. 87 (1998), 67-78), ibid. 103 (2002), 191-200.

[16] —, - Distribution functions of ratio sequences, Publ. Math. Debrecen 58 (2001), 751-778.

[17] R. F. Tichy, Three examples of triangular arrays with optimal discrepancy and linear recurrences, Appl. Fibonacci Numbers 7 (1998), 415-423.

[18] J. T. Tóth, L. Mišík and F. Filip, On some properties of dispersion of block sequences of positive integers, Math. Slovaca 54 (2004), 453-464.

Department of Mathematics

University of J. Selye

Roľníckej školy 1519

94501 Komárno, Slovakia

E-mail: filip.ferdinand@selyeuni.sk
Department of Mathematics University of Ostrava 30. dubna 22

70103 Ostrava 1, Czech Republic E-mail: ladislav.misik@osu.cz janos.toth@osu.cz 